(2) Open Access Full Text Article

\title{
Impact of streptozotocin on altering normal glucose homeostasis during insulin testing in diabetic rats compared to normoglycemic rats
}

This article was published in the following Dove Press journal:

Drug Design, Development and Therapy

5 May 2015

Number of times this article has been viewed

\author{
Nidal A Qinna' \\ Adnan A Badwan² \\ 'Department of Pharmacology \\ and Biomedical Sciences, Faculty \\ of Pharmacy and Medical Sciences, \\ University of Petra, ${ }^{2}$ Research and \\ Innovation Centre, The Jordanian \\ Pharmaceutical Manufacturing Co. Plc. \\ (JPM), Amman, Jordan
}

\begin{abstract}
Streptozotocin (STZ) is currently the most used diabetogenic agent in testing insulin and new antidiabetic drugs in animals. Due to the toxic and disruptive nature of STZ on organs, apart from pancreas, involved in preserving the body's normal glucose homeostasis, this study aims to reassess the action of STZ in inducing different glucose response states in diabetic rats while testing insulin. Diabetic Sprague-Dawley rats induced with STZ were classified according to their initial blood glucose levels into stages. The effect of randomizing rats in such a manner was investigated for the severity of interrupting normal liver, pancreas, and kidney functions. Pharmacokinetic and pharmacodynamic actions of subcutaneously injected insulin in diabetic and nondiabetic rats were compared. Interruption of glucose homeostasis by STZ was challenged by single and repeated administrations of injected insulin and oral glucose to diabetic rats. In diabetic rats with high glucose $(451-750 \mathrm{mg} / \mathrm{dL})$, noticeable changes were seen in the liver and kidney functions compared to rats with lower basal glucose levels. Increased serum levels of recombinant human insulin were clearly indicated by a significant increase in the calculated maximum serum concentration and area under the concentrationtime curve. Reversion of serum glucose levels to normal levels pre- and postinsulin and oral glucose administrations to STZ diabetic rats were found to be variable. In conclusion, diabetic animals were more responsive to insulin than nondiabetic animals. STZ was capable of inducing different levels of normal glucose homeostasis disruption in rats. Both pharmacokinetic and pharmacodynamic actions of insulin were altered when different initial blood glucose levels of STZ diabetic rats were selected for testing. Such findings emphasize the importance of selecting predefined and unified glucose levels when using STZ as a diabetogenic agent in experimental protocols evaluating new antidiabetic agents and insulin delivery systems.
\end{abstract}

Keywords: protein delivery, animal model, diabetes mellitus, experimental, antidiabetic agents, streptozotocin

\section{Introduction}

Several animal models have been developed for studying diabetes mellitus or testing antidiabetic agents. These models include surgical manipulations (pancreatectomy), diet/nutrients-induced diabetes in animals, and genetic manipulations (spontaneous and transgenic/knockout diabetic animals) in various animal species to induce both types of diabetes. ${ }^{1}$ Nevertheless, the most common method for inducing diabetes in animals is the use of diabetogenic drugs that include alloxan monohydrate, ${ }^{2,3}$ streptozotocin (STZ) with or without nicotinamide, ${ }^{4,5}$ ferric nitrilotriacetate,${ }^{6}$ dithizone, ${ }^{7}$ and many other chemicals. ${ }^{1,8}$

According to the literature, STZ is considered as the most used diabetogenic compound for inducing diabetes in experimental animal models. ${ }^{9} \mathrm{STZ}$ is an $N$-nitroso
Correspondence: Nidal A Qinna Department of Pharmacology and Biomedical Sciences, Faculty of Pharmacy and Medical Sciences, University of Petra, Amman, Airport Road, PO Box 961343 Amman II 196, Jordan

Tel +962 65799555

Fax+962 6572764 I

Email nqinna@uop.edu.jo 
derivative of glucosamine extracted from Streptomyces acromogenes. STZ is a pancreatic-selective $\beta$-cell toxin that induces random and rapid and irreversible necrosis of $\beta$ cells. ${ }^{4,10}$ Its $\beta$-cell specificity is mainly the result of selective cellular uptake and accumulation. ${ }^{11}$ In addition to its ability to induce insulin-dependent diabetes mellitus (type 1) by complete removal or destruction of pancreatic $\beta$ cells, STZ was also found capable of developing peripheral insulin resistance or impairing insulin secretion from these cells. Such effects are usually sufficient to induce noninsulin-dependent diabetes mellitus (type 2) in animals.

Many reports indicated that STZ is capable of producing mild to severe types of diabetes that vary according to the selected dose, strain, and age of animals, nutritional status, and route of administration, along with other factors. ${ }^{9,10,12,13}$ Therefore, researchers are obliged to custom-design their own protocols of inducing diabetes with STZ according to each study needs.

Previously, the stage or severity of diabetes induction in animals was mainly judged by achieving a state of hyperglycemia that was described as an increase in the calculated baseline of glucose level post STZ treatment. ${ }^{14,15}$ However, the extent at which the glucose level should be accepted in such studies was not addressed. Generally, rats having initial glucose level above $200 \mathrm{mg} / \mathrm{dL}$ were considered hyperglycemic and included in testing. Nevertheless, such inclusion criteria would allow higher initial glucose levels to be included indiscriminately during studies. This leaves the influence of variation in blood glucose levels following diabetes induction in rats not fully explored. Such variations in glucose baselines could indicate the presence of different levels of glucose homeostasis disruption, which may affect the results of testing antidiabetic agents.

Consequently, the aim of the current investigation is to highlight the impact of STZ diabetes induction on generating different glucose levels in Sprague-Dawley (SD) rats and to find out the differences in the response of glucose homeostasis level to insulin delivered in diabetic and nondiabetic states.

\section{Materials and methods Materials}

Recombinant human (rh) insulin powder was purchased from Biocon Company (Bangalore, India). Calbiochem ${ }^{\circledR}$ STZ was obtained from Merck Millipore Company (Billerica, MA, USA). D-(+)-Glucose, citric acid monohydrate, trisodium citrate dihydrate, and Tris(hydroxymethyl)aminomethane were purchased from Sigma-Aldrich (St Louis, MO, USA). Ten percent neutral buffered formalin was purchased from Merck
KGaA (Darmstadt, Germany). Purified water was provided by the Jordanian Pharmaceutical Manufacturing Company (Naor, Jordan). All chemicals were used as received without any further purification.

\section{Animal handling}

Adult male SD rats with an average weight of $220 \pm 20 \mathrm{~g}$ were purchased from Yarmouk University (Irbid, Jordan) and accommodated at the University of Petra's Animal House Unit under constant temperature $\left(21^{\circ} \mathrm{C}-23^{\circ} \mathrm{C}\right)$, humidity (55\%-65\%), and photoperiod light cycle (12 hours light/ dark). All rats were acclimatized for 10 days before experimenting day and received standard rat chow (Jordan Feed Co. Ltd, Amman, Jordan) and tap water. All animal experiments were performed in compliance with the University of Petra Animal Care Guideline which adapts Federation of European Laboratory Animal Science Association guidelines. The study protocol (SUG/13/55) was approved by the Ethical Committee of the Jordanian Pharmaceutical Manufacturing Company.

\section{Induction of diabetes using STZ}

Freshly dissolved STZ in $0.1 \mathrm{M}$ citrate buffer ( $\mathrm{pH} 4.5$ ) was used to induce diabetes in male SD rats. Two doses of $80 \mathrm{mg} / \mathrm{kg}$ were injected intraperitoneally in the rats over 2 days. On the third day post the first dose of STZ, initial blood glucose levels were determined for the 18-hour fasted rats (access to water ad libitum) by measuring their blood glucose concentrations in blood samples obtained from the tails using a blood glucose meter (GlucoDrTM, All Medicus Co. Ltd, Gyeonggi, Korea). Only rats with a basal blood glucose level above $200 \mathrm{mg} / \mathrm{dL}$ were considered diabetic unless otherwise stated. Occasionally, a single dose of $80 \mathrm{mg} / \mathrm{kg}$ STZ was used to induce diabetes in rats as described above to allow additional classification of blood glucose levels.

\section{Biochemical assays of liver and kidney functions}

Three groups of rats were assigned to receive blank injections (without STZ), a single-dose STZ, or two doses of STZ. On the third day post STZ administration, fasted rats were arbitrarily classified into three stages (groups): Stage 0 of nondiabetic rats having glucose levels $<200 \mathrm{mg} / \mathrm{dL}$, Stage 1 of diabetic rats with glucose levels between $200 \mathrm{mg} / \mathrm{dL}$ and $450 \mathrm{mg} / \mathrm{dL}$, and Stage 2 of diabetic rats with blood glucose levels between $451 \mathrm{mg} / \mathrm{dL}$ and $750 \mathrm{mg} / \mathrm{dL}$. Each group consisted of 10 rats. Immediately after glucose determinations, all rats were euthanized by high dose of inhaled ether. Blood samples were directly withdrawn from the heart chambers and collected into nonheparinized tubes and allowed to clot at room 
temperature and then centrifuged at 4,000 rpm $(3,436 \times g)$ for 5 minutes (accuSpin ${ }^{\text {TM }} 3$ centrifuge, Fisher Scientific, Schwerte, Germany). Serum samples were collected and stored at $-20^{\circ} \mathrm{C}$ until analysis. A drop of blood obtained directly from each heart was used to confirm blood glucose levels and compared to tail glucose determinations. The stored serum samples were later assayed for the following parameters: urea, creatinine, alanine aminotransferase, aspartate aminotransferase, alkaline phosphatase, gamma-glutamyl transferase, and albumin. The tests described earlier were conducted in the Laboratory of Al Mahabba Hospital (Madaba, Jordan) using standard procedures for animal samples testing.

\section{Procedures for histopathology}

Gross appearance and color change in the internal organs were checked for the sacrificed animals using a magnifying glass. Biopsies from liver, kidney, and pancreas of diabetic rats and nondiabetic rats were immediately collected after sacrifice. The tissues were instantly fixed in $10 \%$ phosphatebuffered formalin and embedded in paraffin blocks and sectioned into $5-\mu \mathrm{m}$ thin sections. Sections were stained with hematoxylin and eosin and examined under a light microscope (Nikon, Tokyo, Japan).

\section{Serum insulin pharmacokinetics in STZ diabetic rats}

$\mathrm{SD}$ rats were randomized into two groups ( $\mathrm{n}=40$ per group): a nondiabetic group and a diabetic group. Recombinant human insulin solution was freshly prepared in Tris buffer $(\mathrm{pH} 7)$ as described by Elsayed et al ${ }^{15}$ and injected subcutaneously at a dose of $4 \mathrm{IU} / \mathrm{kg}$ into the scruff of each rat neck in both groups. In an interim design, nonheparinized blood samples ( $n=8$ per time interval per group) were collected directly from the heart chambers of euthanized rats at 0 time (prior insulin injection), 15 minutes, 30 minutes, 60 minutes, and 120 minutes insulin administration and kept in ice. The serum was then separated by centrifugation at 4,000 $\mathrm{rpm}(3,436 \times \mathrm{g})$ for 5 minutes and stored at $-20^{\circ} \mathrm{C}$ until analysis.

A chemiluminescent microparticle immunoassay was used to determine serum insulin levels (in $\mu \mathrm{U} / \mathrm{mL}$ ) utilizing ARCHITECT $i 1000 \mathrm{SR}^{\circledR}$ immunoassay analyzer (Abbot Laboratories, Abbott Park, IL, USA). Before analysis, manual dilution (1:10) was performed for all serum samples by adding $20 \mu \mathrm{L}$ of serum sample to $180 \mu \mathrm{L}$ of ARCHITECT Insulin Calibrator A according to the manufacturer's instructions. Serum insulin levels versus time were plotted, and the maximum serum concentration $\left(C_{\max }\right)$, time of maximum serum concentration, and the area under the concentration-time curve were calculated for each profile following the trapezoidal rule.

\section{Insulin administration to different levels of STZ diabetic rats}

Acclimatized SD rats $(n=50)$ were treated with a single dose of $80 \mathrm{mg} / \mathrm{kg}$ STZ. After 72 hours, blood glucose level was determined for each rat from the tail. STZ-treated rats were classified into three groups according to their glucose levels $(n=7)$. The first group consisted of rats with glucose levels less than $150 \mathrm{mg} / \mathrm{dL}$, while the second group consisted of rats with glucose levels between $150 \mathrm{mg} / \mathrm{dL}$ and $250 \mathrm{mg} / \mathrm{dL}$, and a third diabetic group with glucose determinations of above $400 \mathrm{mg} / \mathrm{dL}$. All rats were injected subcutaneously with $1 \mathrm{IU} / \mathrm{kg}$ insulin. Blood glucose levels were determined at 0.5 hour, 1 hour, 2 hours, 3 hours, 4 hours, 5 hours, and 6 hours after insulin administration. Glucose profiles were plotted for each group as the means of percentage glucose level change from the initial glucose reading of each rat (represented as \%glucose level) versus time of subsequent glucose determinations.

\section{Effect of insulin dose on STZ diabetic and nondiabetic rats}

In this study, different doses of insulin, namely, $1 \mathrm{IU} / \mathrm{kg}$, $2 \mathrm{IU} / \mathrm{kg}, 4 \mathrm{IU} / \mathrm{kg}$, and $6 \mathrm{IU} / \mathrm{kg}$ were injected subcutaneously into nondiabetic rats and STZ diabetic rats ( $\mathrm{n}=10$ per group). After initial glucose determinations, blood glucose measurements were performed at 0.5 hour, 1 hour, 2 hours, 3 hours, 4 hours, 5 hours, and 6 hours postinsulin administration followed by plotting the \%glucose levels against time for all groups.

\section{Effect of repeated injections of insulin on STZ diabetic and nondiabetic rats}

Three groups of nondiabetic rats and three groups of STZ diabetic rats were prepared ( $n=10$ per group), and the initial blood glucose values were determined. Each group received repeated insulin injections of an assigned dose ( $0.5 \mathrm{IU} / \mathrm{kg}, 1 \mathrm{IU} / \mathrm{kg}$, or $2 \mathrm{IU} / \mathrm{kg}$ insulin subcutaneously) every 3 hours. Blood glucose measurements from the tails were performed as described earlier at different time intervals (Figure 5).

\section{Glucose tolerance test protocol on STZ diabetic rats}

STZ diabetic rats (glucose $>200 \mathrm{mg} / \mathrm{dL}$ ) were induced by $\mathrm{STZ}$ and randomized into four groups $(\mathrm{n}=7)$. All groups were administered three doses oral glucose solution $(250 \mathrm{mg} / \mathrm{kg} / \mathrm{h})$. While receiving the third oral glucose dose, initial blood glucose levels were measured for all rats followed by injecting one group subcutaneously with an insulin-free solution; while the other three groups were injected subcutaneously with 
$1 \mathrm{IU} / \mathrm{kg}, 2 \mathrm{IU} / \mathrm{kg}$, and $4 \mathrm{IU} / \mathrm{kg}$ insulin, respectively. Blood glucose determinations were performed at different time intervals as illustrated in Figure 6.

\section{Effect of oral glucose administration following repeated insulin injections on STZ diabetic rats}

In this study, three groups of STZ diabetic rats were randomized $(n=10)$. Each group was assigned to receive initially repeated doses of subcutaneous insulin $(0.5 \mathrm{IU} / \mathrm{kg}, 0.75 \mathrm{IU} / \mathrm{kg}$, and $1 \mathrm{IU} / \mathrm{kg}$ ) every 2 hours followed by oral glucose administration (50 mg per rat) 6 hours post study initiation. Blood glucose determinations were performed every 30 minutes during insulin administration and hourly post oral glucose administration.

\section{Statistics}

One-way analysis of variance was used to compare the differences between groups while multiple comparisons were performed by Tukey's post hoc test (SPSS 17 statistical package, SPSS Inc., Chicago, IL, USA). A probability value $(P)$ of $<0.05$ was considered as the minimum level of statistical significance. All data are expressed as means \pm standard error of mean.

\section{Results}

\section{Effect of STZ diabetes induction on the liver and kidney functions}

Blood biochemistry parameters of selected liver and kidney function tests determined for rats with different stages of initial glucose levels are listed in Table 1. Changes in all liver and kidney function tests were observed when comparing nondiabetic rats (Stage 0: glucose $<200 \mathrm{mg} / \mathrm{dL}$ ) with Stage 2 diabetic rats (glucose: 451-750 mg/dL) except for albumin values. However, it was found that creatinine, aspartate aminotransferase, gamma-glutamyl transferase, and again albumin levels were found not essentially altered when comparing nondiabetic rats with Stage 1 diabetic rats (glucose: $200-450 \mathrm{mg} / \mathrm{dL}$ ). The results of diabetes induction were confirmed by both gross organs examination and histology of liver, kidneys, and pancreas of STZ-treated rats (Figure 1). The excised diabetic rats' livers were visually seen to have more yellowish-brown color, while in kidneys and pancreas, nondetectable gross changes in color, size, or cyst formation were observed. The histology of liver sections obtained from diabetic rats showed clear diffused hepatic steatosis with the loss of usual concentric arrangements of hepatocytes and portal vessels (Figure 1B). The sinusoids were also congested. Sections from diabetic kidneys revealed tubular atrophy and increased vacuolization of renal tubular epithelial cells as illustrated in Figure 1D. Sections of diabetic pancreas, on the other hand, detected reduction in the number of normal islets. The endochylema of islets appeared to have granular degeneration and serious necrosis (Figure 1F).

\section{Impact of STZ diabetes induction on the pharmacokinetics of insulin}

Figure 2 illustrates the pharmacokinetic profiles of insulin injected subcutaneously in STZ diabetic and nondiabetic rats. Injecting a high dose of insulin ( $4 \mathrm{IU} / \mathrm{kg}$ ), as a serum detectable dose without inducing hypoglycemia shocks, increased the $C_{\max }$ value of diabetic rats to $1,591 \mu \mathrm{U} / \mathrm{mL}$ compared to nondiabetic rats, where the $C_{\max }$ reached $1,168 \mu \mathrm{U} / \mathrm{mL}$. No changes in the time of maximum serum concentration values (15 minutes) were detected between different groups. The calculated area under the concentration-time curve of diabetic rats was doubled compared to that of nondiabetic rats.

Table I Selected liver and kidney biochemical values of rats having different stages of initial glucose levels

\begin{tabular}{llll}
\hline Biochemical test & \multicolumn{2}{l}{ Stage of diabetes (glucose level, $\mathbf{~ m g / d L )}$} & Stage 2 (45 I-750) \\
\cline { 2 - 4 } & Stage 0 (<200) & Stage I (200-450) & $628 \pm 26.4^{* *}$ \\
\hline Glucose (tail, mg/dL) & $84 \pm 2.3$ & $397 \pm 12 . I^{* *}$ & $668 \pm 34.5^{* *}$ \\
Glucose (heart, mg/dL) & $88.5 \pm 4.1$ & $439 \pm 20.9^{* *}$ & $148.8 \pm 9.3^{* *}$ \\
Urea (mg/dL) & $39.9 \pm 2.2$ & $70.7 \pm 10.2^{*}$ & $1.6 \pm 0.2^{* *}$ \\
Creatinine (mg/dL) & $0.5 \pm 0.0$ & $1.1 \pm 0.3$ & $80.1 \pm 8 . I^{*}$ \\
ALT (IU/L) & $37.2 \pm 3$ & $88 \pm 18.8^{*}$ & $151.8 \pm 22.9^{*}$ \\
AST (IU/L) & $90.9 \pm 7$ & $131.4 \pm 23.1$ & $322.7 \pm 25.5^{* *}$ \\
ALP (IU/L) & $89.5 \pm 27.6$ & $247 \pm 47.5^{* *}$ & $9.5 \pm 4 . I^{*}$ \\
GGT (IU/L) & $1.8 \pm 0.5$ & $3.4 \pm 0.8$ & $4.9 \pm 0.2$ \\
Albumin (g/dL) & $4.8 \pm 0.2$ & $4.5 \pm 0.3$ & \\
\hline N
\end{tabular}

Notes: Each value represents mean \pm standard error of mean, $n=10$. Statistical analysis was done by comparing Stage 0 (nondiabetic rats) with the other stages of diabetes. $* P<0.05, * * P<0.01$.

Abbreviations: ALT, alanine aminotransferase; AST, aspartate aminotransferase; ALP, alkaline phosphatase; GGT, gamma-glutamyl transferase. 

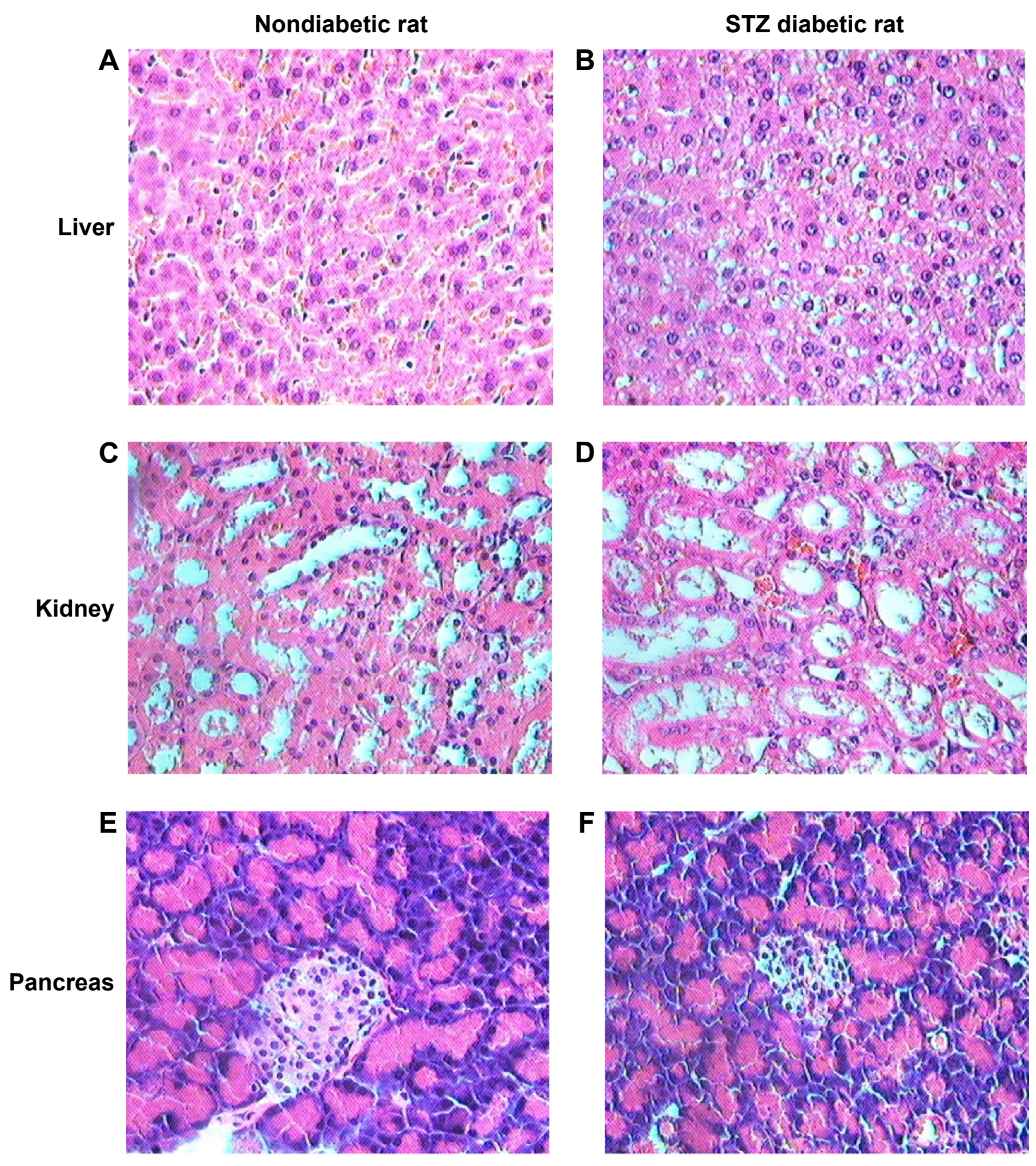

Figure I Representative photomicrographs of hematoxylin and eosin-stained liver (A, B), kidney (C, D), and pancreas (E, F) of normal nondiabetic rats (first column) and STZ-induced diabetic rats (second column). Original magnification $\times 400$.

Abbreviation: STZ, streptozotocin.

Such increase in the circulating insulin might reflect some kind of lack of insulin degradation in the liver of diabetic animals and lower elimination rates from the deteriorated kidneys.

\section{Effect of insulin on STZ diabetic rats with different levels of blood glucose}

Rats treated with a single dose of STZ $(80 \mathrm{mg} / \mathrm{kg})$ were randomized into three groups according to their measured initial blood glucose levels as illustrated in Figure 3. It was noted that the effect of injecting $1 \mathrm{IU} / \mathrm{kg}$ insulin to STZ-treated rats on reducing blood glucose levels was more pronounced in the group of high initial glucose level (glucose $>400 \mathrm{mg} / \mathrm{dL}$ ) compared to the other groups. Tukey's post hoc analysis revealed that the reduction in the percentage of glucose level in this group reached $41 \% \pm 7 \%$ and was highly significant $(P<0.01)$ compared to $83 \% \pm 6 \%$ in the group of glucose $<150 \mathrm{mg} / \mathrm{dL}$ at 2 hours after insulin injection. Therefore, the higher the initial glucose level of the grouped rats in testing, the higher the action of the tested insulin was reported. 


\begin{tabular}{lll}
\hline & Nondiabetic & STZ diabetic \\
\hline AUC $^{*}$ & 45,254 & 92,062 \\
$C_{\max }$ & 1,168 & 1,591 \\
$T_{\max }$ & 15 & 15 \\
\hline
\end{tabular}

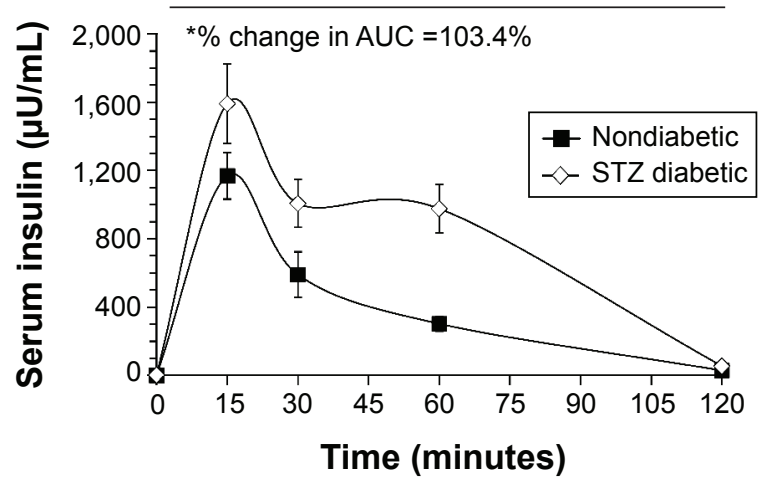

Figure 2 Serum recombinant human insulin $(\mu \mathrm{U} / \mathrm{mL})$ pharmacokinetic profiles of nondiabetic rats and STZ-induced diabetic rats injected with insulin subcutaneously at a dose of $4 \mathrm{lU} / \mathrm{kg}$.

Notes: Interim blood sample collection was performed at different times. Each data point represents the mean \pm standard error of mean, $n=8$ measurements. The upper table summarizes the determined area under the concentration-time curve (AUC), maximum serum concentration $\left(C_{\max }\right)$, and time of maximum serum concentration $\left(T_{\max }\right)$ values of the presented charts.

Abbreviation: STZ, streptozotocin.

Whether this is simply due to changes in its concentration or its bioactivity, such as the activity of insulin, needs to be further explored to eliminate error during experimentation.

\section{Effect of insulin dose on blood glucose levels of STZ diabetic rats compared}

\section{to nondiabetic rats}

The effect of injecting different doses of insulin, namely, $1 \mathrm{IU} / \mathrm{kg}, 2 \mathrm{IU} / \mathrm{kg}, 4 \mathrm{IU} / \mathrm{kg}$, and $6 \mathrm{IU} / \mathrm{kg}$, on changing blood

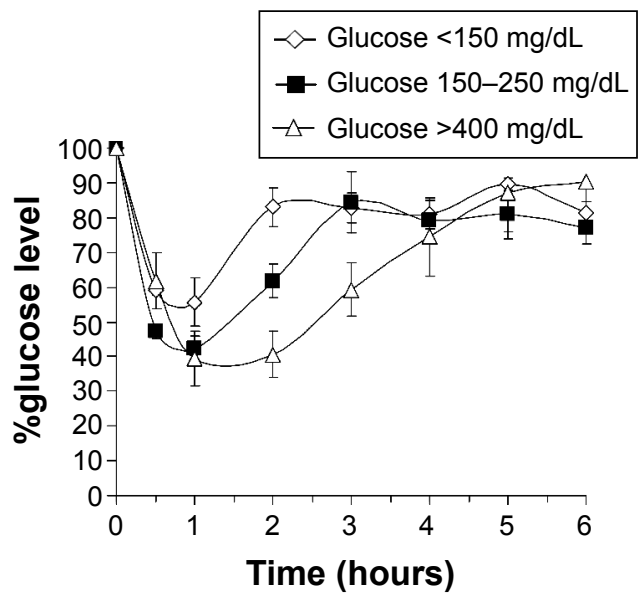

Figure 3 Changes in \%glucose levels versus time profiles of STZ diabetic rats having different initial glucose levels and injected subcutaneously with insulin (I IU/kg). Notes: The diabetic rats were classified into three stages according to their blood glucose. The difference between groups was significant at time intervals up to 4 hours $(P<0.05)$. Data points are expressed as mean \pm standard error of mean $(n=7)$.

Abbreviation: STZ, streptozotocin. glucose levels of STZ diabetic rats compared to nondiabetic rats were pronounced (Figure 4). High differences, as revealed by one-way analysis of variance, between means of glucose levels were obtained when nondiabetic and diabetic groups were compared $(P<0.001)$. Injecting different doses of insulin to diabetic rats (Figure 4B) was more effective in reducing blood glucose levels than in nondiabetic rats (Figure 4A). For example, the minimum percentage of glucose level reached following 2 hours of treating diabetic rats with $6 \mathrm{IU} / \mathrm{kg}$ of insulin was $13.4 \% \pm 1 \%$ compared to $40.7 \% \pm 4 \%$ in nondiabetic rats. The results also indicated that increasing the dose of injected insulin above $2 \mathrm{IU} / \mathrm{kg}$ did not induce any further reductions in blood glucose levels in both examined groups $(P>0.05)$. Furthermore, it was noted that blood glucose levels failed to return to the normal baseline after injecting high doses of insulin (4 IU/kg and $6 \mathrm{IU} / \mathrm{kg}$ ) to
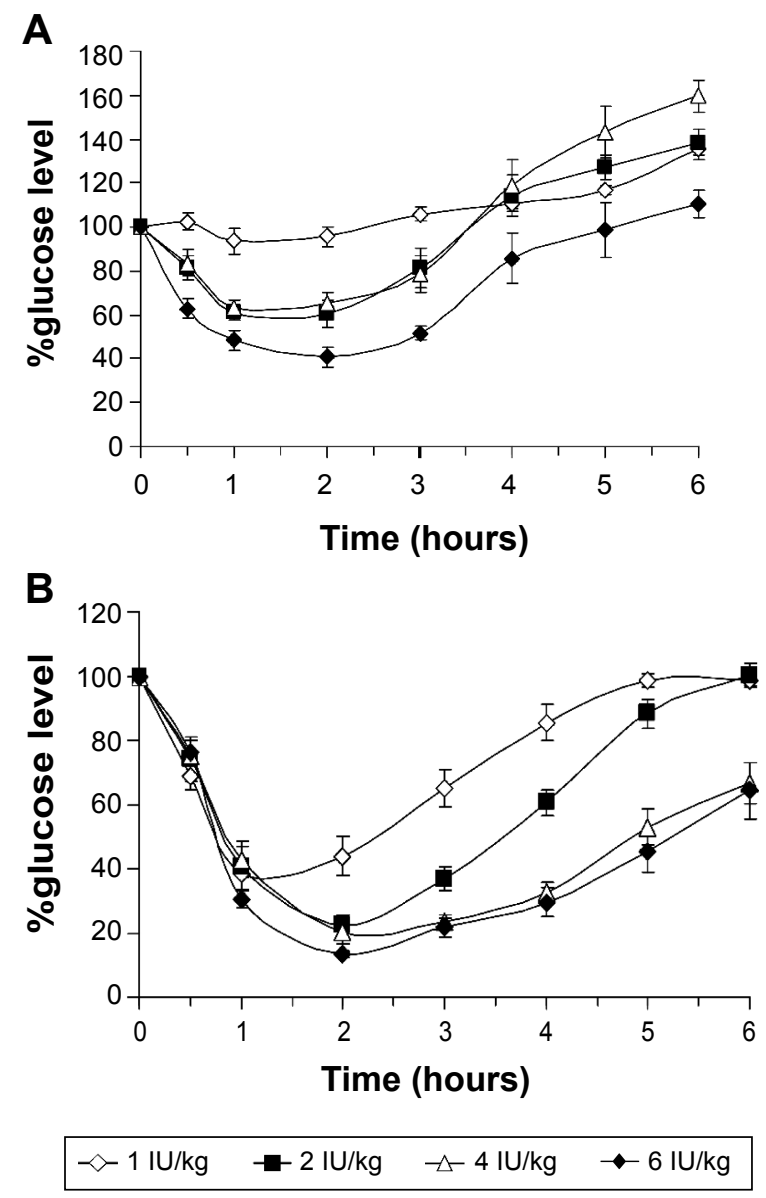

Figure 4 Changes in \%glucose levels versus time profiles of normal nondiabetic rats (A) compared to STZ diabetic rats (B).

Notes: Rats were injected subcutaneously with I IU/kg, $2 \mathrm{lU} / \mathrm{kg}, 4 \mathrm{lU} / \mathrm{kg}$, and $6 \mathrm{IU} /$ $\mathrm{kg}$, and blood glucose measurements were performed at different time intervals. The difference (analysis of variance) between nondiabetic rats and STZ diabetic rats was found to be significant $(P<0.05)$. Data points are expressed as mean \pm standard error of mean $(n=10)$.

Abbreviation: STZ, streptozotocin. 
diabetic rats. This might indicate the availability of excess insulin in blood at these high doses and show that its activity against blood glucose is attained.

\section{Challenging glucose homeostasis with repeated insulin administration in diabetic and nondiabetic rats}

Repeated doses of insulin were injected to nondiabetic rats (Figure 5A) and STZ diabetic rats (Figure 5B) at 0 hour, 3 hours, 6 hours, and 9 hours post initial glucose level determinations. The selected doses of insulin were reduced to $0.5 \mathrm{IU} / \mathrm{kg}, 1 \mathrm{IU} / \mathrm{kg}$, and $2 \mathrm{IU} / \mathrm{kg}$ in order to prevent the treated rats from experiencing hypoglycemic shocks. Once again, the results indicated a more pronounced effect of insulin on reducing blood glucose levels in diabetic rats than nondiabetic rats. Due to the presence of normal glucose homeostasis, nondiabetic rats have resisted the reduction of glucose levels as indicated from the observed fluctuation of glucose levels pre- and post-injecting insulin. In this study, a decision was made to terminate the 2-IU/kg insulin administration and glucose determinations in both nondiabetic and diabetic groups due to the development of persistent hypoglycemia in rats that rendered them immobile and moribund with a reported death rate of $70 \%$ and $50 \%$, respectively, in both groups.

\section{Glucose tolerance test on STZ diabetic rats}

Figure 6 illustrates the glucose level profiles of STZ diabetic and nondiabetic rats initially administered with three doses of glucose solution $(250 \mathrm{mg} / \mathrm{h} / \mathrm{rat})$ followed by challenging

A

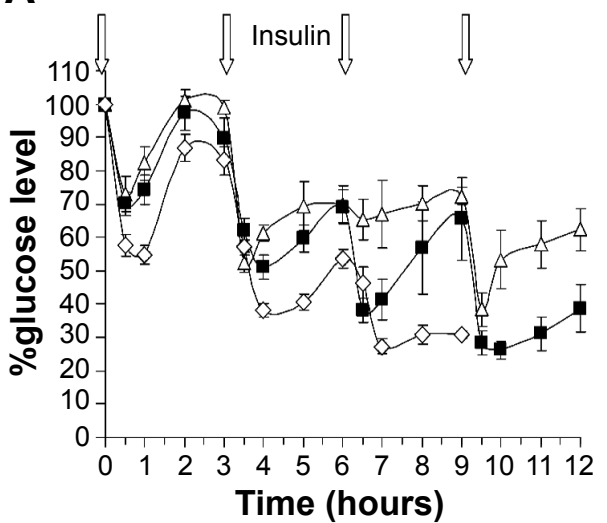

the blood glucose levels with different doses of subcutaneous insulin. Blood glucose levels were increased in all groups due to oral glucose administration. However, glucose increase, due to the third oral glucose dose, above the basal level was more pronounced in the nondiabetic rats (Figure 6A) compared to STZ diabetic rats (Figure 6B). Glucose levels were highly reduced following insulin administration compared to noninsulin (placebo)-treated rats in both groups. The return of each glucose level to its corresponding baseline was highly dependent on the injected insulin dose. It was found that lowering the injected insulin dose resulted in faster glucose level returns. Statistically, overall differences between means of glucose levels in both groups were revealed significant by one-way analysis of variance test $(P<0.01)$. Nevertheless, multiple comparisons using Tukey's post hoc analysis revealed no significant differences between the blood glucose profiles of rats treated with $1-\mathrm{IU} / \mathrm{kg}$ compared to the $2-\mathrm{IU} / \mathrm{kg}$ insulin-injected rats.

\section{Effect of repeated insulin administration on the glucose homeostasis of STZ diabetic rats}

Results of challenging blood glucose levels of diabetic rats with oral glucose administration post repeated doses of insulin are illustrated in Figure 7. Lower doses of insulin, namely, $0.5 \mathrm{IU} / \mathrm{kg}, 0.75 \mathrm{IU} / \mathrm{kg}$, and $1 \mathrm{IU} / \mathrm{kg}$, were selected since we have reduced in this study the injection time interval to 1 hour and increased the number of insulin injections to six per group. Glucose levels were forced to decrease in all groups at the same rate in the first 3 hours due to repeated

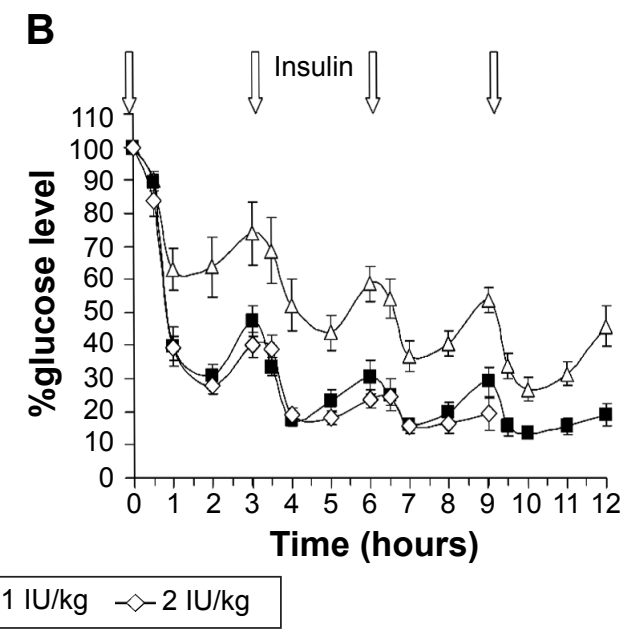

Figure $\mathbf{5}$ Changes in \%glucose levels versus time profiles of normal nondiabetic rats (A) compared to STZ diabetic rats (B).

Notes: Repeated doses of insulin ( $0.5 \mathrm{lU} / \mathrm{kg}, \mathrm{I} \mathrm{IU} / \mathrm{kg}$, and $2 \mathrm{lU} / \mathrm{kg}$ ) were injected subcutaneously in rats at 0 hour, 3 hours, 6 hours, and 9 hours time intervals (arrows). The loss of normal glucose homeostasis was obvious in STZ diabetic rats especially at I- and 2-IU/kg doses. At the highest injected insulin dose (2 IU/kg), glucose measurements were stopped after 9 hours of experimentation in both nondiabetic and diabetic groups due to hypoglycemia. Data points are expressed as mean \pm standard error of mean ( $\mathrm{n}=10$ ). Abbreviation: STZ, streptozotocin. 

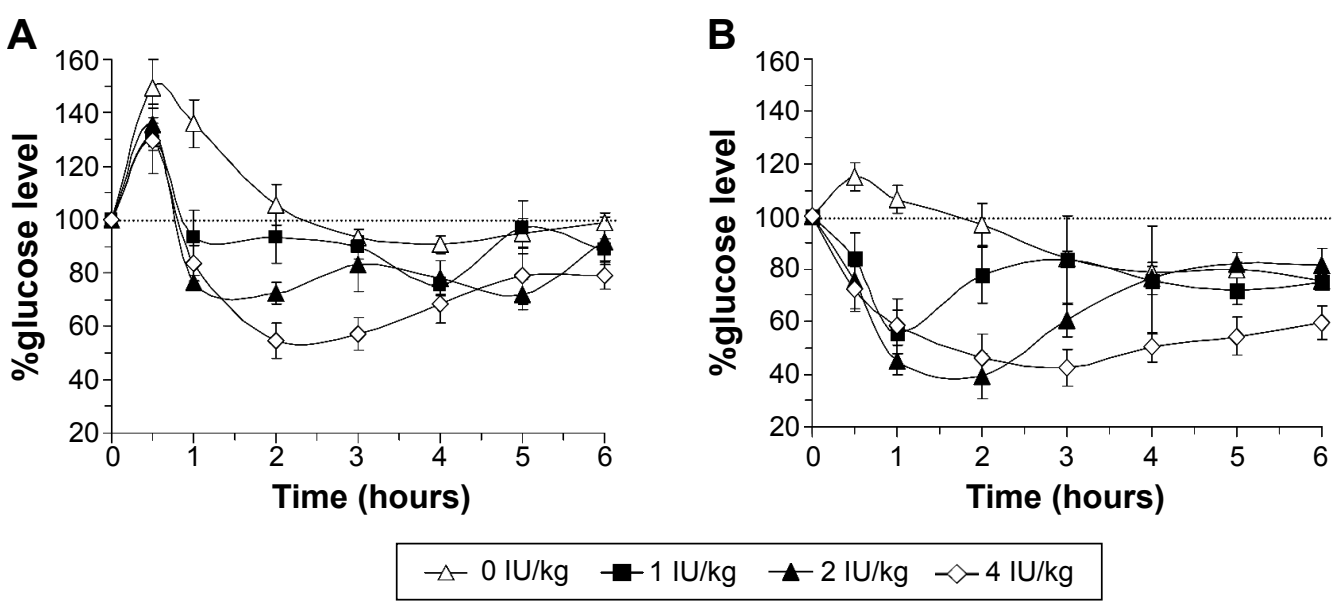

Figure 6 Changes in \%glucose levels versus time profiles of normal nondiabetic rats (A) compared to STZ diabetic rats (B) challenged initially with repeated oral glucose administration (three doses of $250 \mathrm{mg} / \mathrm{h} / \mathrm{rat}$ ) followed by injecting subcutaneously different doses of insulin $(0 \mathrm{IU} / \mathrm{kg}, \mathrm{I} \mathrm{IU} / \mathrm{kg}, 2 \mathrm{IU} / \mathrm{kg}$, and $4 \mathrm{IU} / \mathrm{kg}$ ) simultaneously with the last oral glucose dose.

Notes: Baseline glucose level is illustrated as a dotted line. Data points are expressed as mean \pm standard error of mean ( $n=7)$.

Abbreviation: STZ, streptozotocin.

insulin administration. The differences between groups were found to be statistically insignificant in the first 6 hours $(P>0.05)$, although rats injected with $0.5 \mathrm{IU} / \mathrm{kg}$ insulin showed a pattern of resistance of glucose reduction post the third insulin injection. However, the change was found insignificant when multiple comparison tests were performed. It is observed in Figure 7 that repeated administration of insulin to diabetic rats induced plateaus in the glucose level

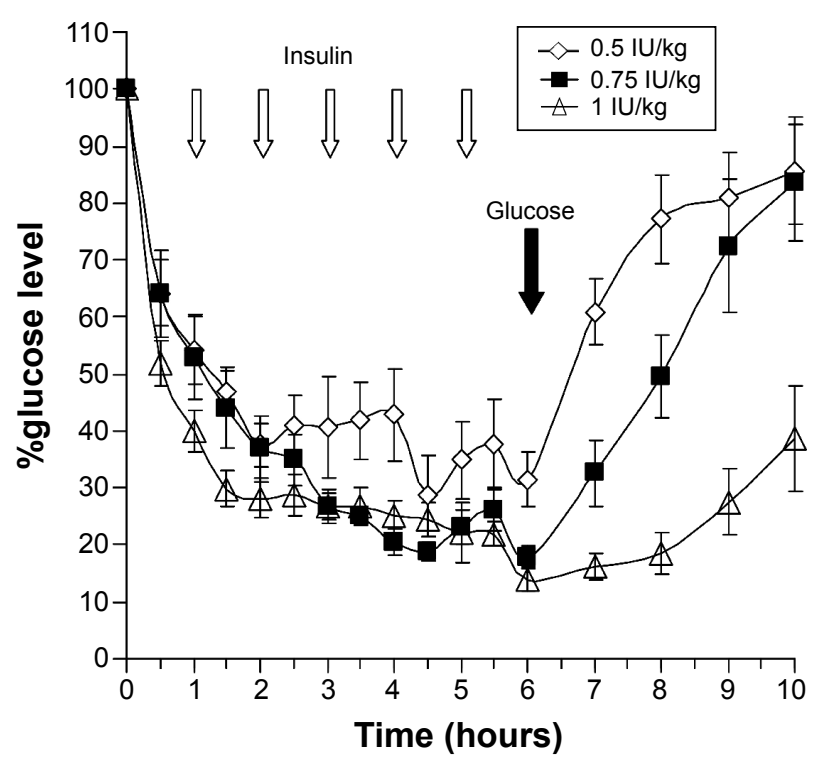

Figure 7 Changes in \%glucose levels versus time profiles of STZ diabetic rats injected initially with repeated doses of insulin $(0.5 \mathrm{IU} / \mathrm{kg}, 0.75 \mathrm{IU} / \mathrm{kg}$, and I IU/kg) every 2 hours (white arrows) followed by oral glucose administration $(50 \mathrm{mg}$ per rat) after 6 hours (black arrow) of blood glucose measurements.

Notes: \%Glucose levels were significantly different after oral glucose administration $(P<0.05)$. Data points are expressed as mean \pm standard error of mean $(n=10)$. Abbreviation: STZ, streptozotocin. profiles. These plateaus were formed between \%glucose levels of $15 \%-25 \%$, which indicated the presence of relative glucose homeostasis that might have protected the tested rats from hypoglycemia. Most importantly, when oral glucose (50 mg per rat) was administered to the insulin-treated rats, all glucose levels increased in a dose-dependent manner and differences between groups were found to be significant $(P<0.001)$. Blood glucose levels increased faster in the $0.5-\mathrm{IU} / \mathrm{kg}$ insulin-treated group compared to the other groups; while it was seen that glucose level increment of $1-\mathrm{IU} / \mathrm{kg}$ insulin-treated group was slow as confirmed by the calculated slope of each profile.

\section{Discussion}

Since the demonstration of the diabetogenic property of STZ by Rakieten et al, ${ }^{4}$ the induction of diabetes with STZ in rodents has been widely considered. STZ is able to pass through any cell membrane that contains glucose transporter (GLUT-2). Such property excludes the pharmacological specificity of this diabetogenic agent in the pancreas. It has been reported by Szkudelski in 2001 that once this drug passes the cell membrane, it causes alkylation of DNA. This damage leads to the depletion of cellular NAD+ and ATP and the formation of superoxide radicals. STZ can also liberate nitric oxide inside the cells that inhibits aconitase activity resulting in further DNA damage. ${ }^{16}$ Indeed such toxic activity is more pronounced in $\beta$ cells in pancreas leaving $\alpha$ and $\gamma$ cells intact. ${ }^{17}$

A considerable cross talk between the liver and kidney has been reported in controlling optimal glucose homeostasis, a 
relationship referred to as hepatorenal glucose reciprocity. ${ }^{18}$ Although hepatotoxicity and nephrotoxicity are both well documented effects of STZ, ${ }^{19,20}$ none of the previous studies related such toxicities with the determined initial blood glucose levels. It is evident that the action of STZ on the body is nonspecific and this implicates a random process in deteriorating the body organs. Indeed, the current work shows that beside the reduction of endogenous insulin secretions due to pancreatic destruction, the exogenous injected insulin was accumulated more in the blood of the diabetic animals. This might be attributed to a decrease in the kidney and liver functions. Such combination of inefficient metabolism and excretion caused the accumulation of the injected insulin in the blood circulation.

In many reported studies, STZ was routinely used to induce a state of hyperglycemia in rats, mainly in SD and Wistar strains, by injecting them with one or more intraperitoneal or intravenous doses of STZ that range from $40 \mathrm{mg} / \mathrm{kg}$ to $100 \mathrm{mg} / \mathrm{kg}$. The optimum dose is left to the investigator's choice. Due to the randomness of the deterioration of different organs involved in glucose homeostasis, the selection of low doses of STZ would not be expected to highlight the pathological changes in the tissues. This explains the lack of changes in the inspected tissues, but still higher doses of STZ would clearly damage the organs involved in insulin secretion, metabolism, and elimination. Consequently, it was found that the deleterious actions on such organs was guaranteed after delivering two consecutive doses of $80 \mathrm{mg} / \mathrm{kg}$ of STZ as illustrated in Figure 1 and Table 1. Moreover, experiments with the higher dose of STZ showed less variation in results.

It was necessary to investigate whether upset of the normal glucose homeostasis by STZ could alter the pharmacokinetics of the injected insulin in diabetic rats compared to nondiabetic rats. As expected, increased levels of serum exogenous insulin in diabetic rats were evident. The $C_{\max }$ has increased, and the area under the concentration-time curve value was doubled in the case of diabetic rats compared to normal rats (Figure 2). This strongly suggests that insulin clearance, due to liver degradation and kidney elimination, was reduced since it was reported that the liver and kidneys are the major organs for insulin turnover in blood. ${ }^{18,21,22}$ Consequently, the effect of insulin in the STZ diabetic rats was expected to be more pronounced due to the presence of a higher amount of bioactive insulin circulating in the blood. Such observation needs further studies.

Pharmacodynamically, the presented results indicated that blood glucose reduction following insulin administration varied between rats classified according to their initial blood glucose levels after inducing rats with a single dose of STZ (Figure 3). Insulin was confirmed to be more efficacious in reducing the blood glucose levels of the highly diabetic rats (glucose $>400 \mathrm{mg} / \mathrm{dL}$ ). During hyperglycemia, the body attempts to compensate normal glucose homeostasis by producing higher amounts of insulin..$^{23}$ Conversely, it has been reported that hyperglycemia can also induce pancreatic $\beta$-cell dysfunction, reduced $\beta$-cell mass, and insulin deficiency. ${ }^{24}$ However, since the presented results indicated that the pancreas was at least partially deteriorated by STZ, it was expected to be unable to produce a sufficient amount of endogenous insulin to cope with the measured high levels of glucose. Furthermore, the increased efficacy of insulin in highly diabetic rats could be attributed to the reduction of insulin degradation, mainly by the liver, compared to nondiabetic rats as indicated pharmacokinetically.

In normal individuals, endogenous insulin is secreted from the pancreas in a pulsatile manner. ${ }^{25}$ Ferrannini and his associates, however, demonstrated that insulin secretion rates are less responsive to changes in glucose levels in diabetic patients compared to normal subjects. ${ }^{26}$ This indicates the loss of normal glucose homeostasis in diabetic patients. In animals, however, the lack of data on glycemic variation during STZ diabetes induction has urged Grant et al to establish a protocol of repeated insulin therapy for STZ diabetic rats to achieve normoglycemia. ${ }^{27}$ The aim of injecting rats with repeated doses of insulin in the present study (Figure 5) was to test the extent of preserving glucose homeostasis in diabetic rats while being challenged with continuous insulin administration. As expected, the STZ diabetic rats injected with high doses of insulin showed more reduction in glucose levels accompanied with presumptive loss of normal glucose homeostasis as indicated by the loss of the normal curve fluctuations pre- and postinsulin administration. However, the disruption of normal glucose homeostasis in diabetic rats, which was supported earlier with the liver, kidney, and pancreas damage, resulted in losing the ability of glucose levels to return to its corresponding baselines especially when $1 \mathrm{IU} / \mathrm{kg}$ and $2 \mathrm{IU} / \mathrm{kg}$ repeated insulin doses were injected.

Loss of normal glucose tolerance is a characteristic effect of STZ. ${ }^{28}$ Evident glucose intolerance in STZ diabetic rats was observed in response to oral glucose administration (Figure 6B). When insulin was administered to the rats, however, a dose-dependent reduction of blood glucose levels was noticed. In line with the described results, the reduction was more pronounced in diabetic rats compared to nondiabetic rats. In an earlier study, however, Wi et $\mathrm{al}^{29}$ infused glucose in STZ diabetic rats over a longer period of 
time (6 hours) and indicated that the hyperglycemia in STZ diabetic rats was mainly due to decreased glucose clearance rather than increased hepatic glucose output, effects that are both related to kidney and liver deterioration, respectively, as we propose.

Normally, insulin has a very short half-life and should not be active for a long period of time in blood. However, by examining the slopes of blood glucose level curves post repetitive doses of insulin, namely, $6.1 \mathrm{IU} / \mathrm{kg}, 17.1 \mathrm{IU} / \mathrm{kg}$, and $12.8 \mathrm{IU} / \mathrm{kg}$ for $1,0.75$, and $0.5 \mathrm{IU} / \mathrm{kg}$, respectively (Figure 7), it is evident that insulin is still active in consuming the administered glucose in a dose-dependent manner. It seems that there is a mechanism involved in controlling such an action. What is the structure of this excess circulating insulin? Is it a complexed structure? How is such a structure handled by the liver? These questions need to be answered. Although high concentration of insulin is circulating the blood, it did not break the glucose homeostasis of the tested STZ diabetic rats in a dramatic way. This may suggest that there is another mechanism controlling the relationship between circulating insulin and glucose levels in plasma. Indeed, a previous work demonstrated that administrating $5 \mathrm{IU} / \mathrm{kg}$ of oral insulin nanoparticles to STZ diabetic rats reduced blood glucose to the same extent as the $50-\mathrm{IU} / \mathrm{kg}$ dose. ${ }^{30}$ This result indicates that there is reservoir of insulin which keeps circulating the blood in its inactive form but ready to be activated in the presence of excessive glucose. Such observation needs further investigation.

\section{Conclusion}

Diabetic animals were more responsive to insulin than nondiabetic animals. This increase in insulin's response might be attributed to the destructive nature of STZ on the liver and kidneys and, therefore, less metabolism and elimination of insulin. Due to the complexity and overlapping nature of both types of diabetes in preserving the body's normal glucose homeostasis, the presented findings also emphasize the importance of selecting proper and unified basal glucose levels when using STZ as a model for inducing experimental diabetic rats. The pharmacokinetic and pharmacodynamic actions of insulin were versatile when different stages of basal glucose levels were selected for experimentation. Since STZ has been reported to induce different types of diabetes mellitus in animals, outlining a predefined glucose level in experimental protocols evaluating drugs used in diabetes is highly recommended. Care must be taken when testing the effects of various antidiabetic agents on STZ diabetic animals due to the influence of STZ on altering normal glucose homeostasis. Indeed, other factors such as liver glycogen, incretins, and amylin levels that also contribute in maintaining normal glucose homeostasis or affecting insulin response should be further explored during experimental STZ diabetes induction.

\section{Acknowledgments}

The authors thank Dr Husam Abu Farsakh for reading the histopathology slides and Mrs Nawzat Al-Jbour for her assistance in insulin solution preparation.

\section{Author contributions}

Both authors contributed in the conception and design of the experiments and acquisition and analysis of data. Dr Qinna drafted the manuscript and Dr Badwan critically revised the article and both authors agree to be accountable for all aspects of the work.

\section{Disclosure}

This project was financially supported by Suwagh Company (Naor, Jordan) and the Deanship of Scientific Research, University of Petra (Amman, Jordan). The authors declare that there are no other conflicts of interest to report.

\section{References}

1. King AJ. The use of animal models in diabetes research. Br J Pharmacol. 2012;166(3):877-894.

2. Lenzen S. The mechanisms of alloxan- and streptozotocin-induced diabetes. Diabetologia. 2008;51(2):216-226.

3. Tyrberg B, Andersson A, Borg LA. Species differences in susceptibility of transplanted and cultured pancreatic islets to the beta-cell toxin alloxan. Gen Comp Endocrinol. 2001;122(3):238-251.

4. Rakieten N, Rakieten ML, Nadkarni MV. Studies on the diabetogenic action of streptozotocin (NSC-37917). Cancer Chemother Rep. 1963;29: 91-98.

5. Szkudelski T. Streptozotocin-nicotinamide-induced diabetes in the rat. Characteristics of the experimental model. Exp Biol Med (Maywood). 2012;237(5):481-490.

6. Awai M, Narasaki M, Yamanoi Y, Seno S. Induction of diabetes in animals by parenteral administration of ferric nitrilotriacetate. A model of experimental hemochromatosis. Am J Pathol. 1979;95(3):663-673.

7. Hansen WA, Christie MR, Kahn R, et al. Supravital dithizone staining in the isolation of human and rat pancreatic islets. Diabetes Res. 1989; 10(2):53-57.

8. Kumar S, Singh R, Vasudeva N, Sharma S. Acute and chronic animal models for the evaluation of anti-diabetic agents. Cardiovasc Diabetol. 2012;11:9.

9. Etuk EU. Animal models for studying diabetes mellitus. Agric Biol J N Am. 2010;1(2):130-134.

10. Hayashi K, Kojima R, Ito M. Strain differences in the diabetogenic activity of streptozotocin in mice. Biol Pharm Bull. 2006;29(6):1110-1119.

11. Tjalve H, Wilander E, Johansson EB. Distribution of labelled streptozotocin in mice: uptake and retention in pancreatic islets. J Endocrinol. 1976;69(3):455-456.

12. Bonner-Weir S, Trent DF, Honey RN, Weir GC. Responses of neonatal rat islets to streptozotocin: limited B-cell regeneration and hyperglycemia. Diabetes. 1981;30(1):64-69.

13. Sakata N, Yoshimatsu G, Tsuchiya H, Egawa S, Unno M. Animal models of diabetes mellitus for islet transplantation. Exp Diabetes Res. 2012;2012:256707. 
14. Elsayed A,Al-RemawiM, Qinna N,FaroukA, Al-Sou'odKA, Badwan AA. Chitosan-sodium lauryl sulfate nanoparticles as a carrier system for the in vivo delivery of oral insulin. AAPS Pharm Sci Tech. 2011; 12(3):958-964.

15. Elsayed A, Remawi MA, Qinna N, Farouk A, Badwan A. Formulation and characterization of an oily-based system for oral delivery of insulin. Eur J Pharm Biopharm. 2009;73(2):269-279.

16. Szkudelski T. The mechanism of alloxan and streptozotocin action in B cells of the rat pancreas. Physiol Res. 2001;50(6):537-546.

17. Deeds MC, Anderson JM, Armstrong AS, et al. Single dose streptozotocininduced diabetes: considerations for study design in islet transplantation models. Lab Anim. 2012;45(3):131-140.

18. Poretsky L. Principles of Diabetes Mellitus. Boston, MA, USA: Springer; 2010.

19. Palm F, Ortsater H, Hansell P, Liss P, Carlsson PO. Differentiating between effects of streptozotocin per se and subsequent hyperglycemia on renal function and metabolism in the streptozotocin-diabetic rat model. Diabetes Metab Res Rev. 2004;20(6):452-459.

20. Rauter AP, Martins A, Borges C, et al. Antihyperglycaemic and protective effects of flavonoids on streptozotocin-induced diabetic rats. Phytother Res. 2010;24(suppl 2):S133-S138.

21. Cañas X, Fernández-López JA, Ardévol A, et al. Rat insulin turnover in vivo. Endocrinology. 1995;136(9):3871-3876.

22. Doherty JJ 2nd, Kay DG, Lai WH, Posner BI, Bergeron JJ. Selective degradation of insulin within rat liver endosomes. J Cell Biol. 1990; 110(1):35-42.
23. Toschi E, Camastra S, Sironi AM, et al. Effect of acute hyperglycemia on insulin secretion in humans. Diabetes. 2002;51(suppl 1) S130-S133.

24. Nichols CG, Remedi MS. The diabetic beta-cell: hyperstimulated vs hyperexcited. Diabetes Obes Metab. 2012;14(suppl 3):129-135.

25. Song SH, Kjems L, Ritzel R, et al. Pulsatile insulin secretion by human pancreatic islets. J Clin Endocrinol Metab. 2002;87(1):213-221.

26. Ferrannini E, Gastaldelli A, Miyazaki Y, Matsuda M, Mari A, DeFronzo RA. Beta-cell function in subjects spanning the range from normal glucose tolerance to overt diabetes: a new analysis. $J$ Clin Endocrinol Metab. 2005;90(1):493-500.

27. Grant CW, Duclos SK, Moran-Paul CM, et al. Development of standardized insulin treatment protocols for spontaneous rodent models of type 1 diabetes. Comp Med. 2012;62(5):381-390.

28. Thibault C, Laury MC, Bailbe D, Ktorza A, Portha B. Effect of prolonged in vivo glucose infusion on insulin secretion in rats with previous glucose intolerance. Endocrinology. 1992;130(5):2521-2527.

29. Wi JK, Kim JK, Youn JH. Reduced glucose clearance as the major determinant of postabsorptive hyperglycemia in diabetic rats. Am J Physiol. 1998;274(2 pt 1):E257-E264.

30. Elsayed A. Oral delivery of insulin: novel approaches. In: Sezer A, editor. Recent Advances in Novel Drug Carrier Systems. InTech: 2012.
Drug Design, Development and Therapy

\section{Publish your work in this journal}

Drug Design, Development and Therapy is an international, peerreviewed open-access journal that spans the spectrum of drug design and development through to clinical applications. Clinical outcomes, patient safety, and programs for the development and effective, safe, and sustained use of medicines are a feature of the journal, which

\section{Dovepress}

has also been accepted for indexing on PubMed Central. The manuscript management system is completely online and includes a very quick and fair peer-review system, which is all easy to use. Visit http://www.dovepress.com/testimonials.php to read real quotes from published authors.

Submit your manuscript here: http://www.dovepress.com/drug-design-development-and-therapy-journal 